\title{
ON THE DETECTION OF WR STARS IN NEARBY GALAXIES
}

\author{
J. LEQUEUX \\ Observatoire de Meudon \\ 91195 Meudon CEDEX, France \\ and Ecole Normale Supérieure \\ M. AZZOPARDI \\ Observatoire de Marseille \\ 13248 Marseille CEDEX, France
}

\begin{abstract}
The different methods for detecting WR stars in nearby galaxies are reviewed and compared. Each one has its own advantages and drawbacks, and they are complementary rather than competing. Both monochromatic filter imaging and field- or single-object spectroscopy are required for an absolutely safe detection of objects near the limit. The results obtained todate are shortly reviewed and perspectives are discussed.
\end{abstract}

\section{Introduction}

Wolf-Rayet stars are very sensitive tracers of metallicity and age of young stellar populations. At the scale of a large galaxy where recent star formation may be considered as having occured more or less uniformly in time, considerations on the late evolution of massive stars predict that the ratio of the number of WR to $O$ or supergiant stars should be a very sensitive function of metallicity (Maeder et al. 1980). In a recent study, Maeder (1990 and this Symposium) shows that the WO/WN/WC ratios and the distribution between WR subtypes also depends on metallicity. In the case of a starburst the number of WRs with respect to that of other massive stars depends also strongly on time since even the most massive stars take a few million years before turning into WR (Arnault et al. 1989; Mas-Hesse and Kunth 1990). Fortunately, those ratios do not seem to depend much on the initial mass function of massive stars.

It is thus of high importance for understanding the formation and evolution of WR stars and for using them as tracers of metallicity and age of young stellar populations to obtain complete, unbiased samples of WR stars in various environments, e.g. in galaxies or portions of galaxies of different types and metallicities, to secure a spectral classification and photometry for all objects in the sample, and to study their binarity. The present review discusses some of the means for achieving this goal. Another problem, which will not be discussed here, is to obtain for comparison estimates of the number of $\mathrm{O}$ stars and of supergiants of various types. I will describe shortly the advantages and inconvenients of the various methods used for detecting WR stars in nearby galaxies: systematic spectroscopy of bright stars, filter monochromatic imaging and field spectroscopy, then discuss the results and their interpretation. A list of WR surveys done with the two last techniques in nearby galaxies is given in Table 1, with the exception of old Magellanic Cloud surveys and of new surveys reported elsewhere in this Symposium.

$$
619
$$

K. A. van der Hucht and B. Hidayat (eds.),

Wolf-Rayet Stars and Interrelations with Other Massive Stars in Galaxies, 619-624.

(C) 1991 IAU. Printed in the Netherlands. 
TABLE 1: Surveys of WR stars in nearby galaxies

\begin{tabular}{|c|c|c|c|c|c|c|c|c|}
\hline Gal. & Method & $\begin{array}{l}\text { Teles. } \\
(\mathrm{m})\end{array}$ & Detec. & $\begin{array}{l}\text { Filter } \\
\lambda(\Delta \lambda) \mathrm{nm}\end{array}$ & $\begin{array}{l}\text { Disp. } \\
\text { A/mm }\end{array}$ & $\begin{array}{l}\text { Limit } \\
\text { mag. }\end{array}$ & $\begin{array}{l}\text { New(tot. }) \\
W R>5 \sigma\end{array}$ & Ref. \\
\hline${ }_{n}^{\text {LMC }}$ & & 0.40 & Phot & $465(12)$ & 15 & & $\begin{array}{l}13(78) \\
4(101)\end{array}$ & $\begin{array}{l}\text { 79AZb } \\
80 A Z\end{array}$ \\
\hline$"$ & $"$ & $"$ & $"$ & $"$ & $"$ & $"$ & $2(105)$ & $85 A Z$ \\
\hline$"$ & $"$ & 1.2 & $"$ & Various & 600 & 19 & 4 & $85 \mathrm{MOR}$ \\
\hline " & Mon.Im. & $"$ & $"$ & $469(7,150)$ & - & $"$ & 2 & \\
\hline " & Field Sp. & $"$ & $"$ & $498(85)$ & 800 & - & $0(2)$ & 90MOR \\
\hline 30Dor & Mon. Im. & 4 & CCD & $\begin{array}{l}465,469(4) \\
470(35)\end{array}$ & - & - & $1(17)$ & $85 \mathrm{MOFb}$ \\
\hline SMC & Field Sp. & 0.40 & Pho & $465(12)$ & 150 & 17.5 & $4(8)$ & $79 A Z a$ \\
\hline M31 & Mon.Im. & 3.6 & Phot & $\begin{array}{l}467(9) \\
450(100)\end{array}$ & - & 21.5 & 17 & 83MOd \\
\hline$"$ & $"$ & $"$ & CCD & 4 filt. (6) & - & 21.5 & 20 & 86MASa \\
\hline$"$ & " & $"$ & Im.tube & e $467,445(90)$ & - & 21.5 & 20 & $87 \mathrm{MOFb}$ \\
\hline$"$ & Field Sp. & $"$ & Phot & $483(95)$ & 2000 & 20 & $15(50)$ & 90MEY \\
\hline $\begin{array}{l}\text { M33 } \\
\text { " }\end{array}$ & Mon.Im. & $\begin{array}{l}2.1 \\
3.6\end{array}$ & $\begin{array}{l}\text { Im.tube } \\
\text { Im.tube }\end{array}$ & $\begin{array}{l}\text { e } 445,467(7) \\
\text { e } 467,445(10)\end{array}$ & $\overline{-}$ & $\begin{array}{l}21: \\
21\end{array}$ & $\begin{array}{l}25 \\
41\end{array}$ & $\begin{array}{l}\text { 72WRA } \\
\text { 83MASb }\end{array}$ \\
\hline " & Field Sp. & 4 & Phot & $420(200)$ & 1500 & 21: & $0(4)$ & $85 \mathrm{BOH}$ \\
\hline$"$ & Mon.Im. & 3.6 & Im.tube & e $467,445(9)$ & - & 21 & $51(115)$ & 87MASb \\
\hline$"$ & Field Sp. & 3.6 & Phot. & $483(95)$ & 2000 & 20 & $0(52)$ & 87LEQ \\
\hline " & Mon.Im. & 2.2 & CCD & 469(3,5)etc. & - & - & $14(59)$ & 90DRI \\
\hline HII reg & Mon.Im. & 3.6 & CCD & $\begin{array}{l}469,465(3) \\
478(7)\end{array}$ & - & - & $10(26)$ & $"$ \\
\hline $\begin{array}{l}\text { NGC } \\
6822 \\
" ~\end{array}$ & $\begin{array}{l}\text { Field Sp. } \\
\text { Mon.Im. } \\
\text { " }\end{array}$ & $\begin{array}{l}3.6 \\
2.2,3.6 \\
4\end{array}$ & $\begin{array}{l}\text { Phot } \\
\text { Im.tube } \\
\text { CCD }\end{array}$ & $\begin{array}{l}483(95) \\
\text { e } 467(9) \text { etc. } \\
\text { various }\end{array}$ & $\begin{array}{l}2200 \\
- \\
-\end{array}$ & $\begin{array}{l}20 \\
21.5 \\
21.5\end{array}$ & $\begin{array}{l}1 \\
0 \\
3(4)\end{array}$ & $\begin{array}{l}\text { 83WE } \\
83 \mathrm{MOd} \\
85 \mathrm{AR}\end{array}$ \\
\hline $\begin{array}{l}\text { IC } \\
1613\end{array}$ & $\begin{array}{l}\text { Mon.Im. } \\
\text { Field Sp. }\end{array}$ & $\begin{array}{l}4 \\
3.6\end{array}$ & $\begin{array}{l}\text { CCD } \\
\text { Phot }\end{array}$ & $\begin{array}{l}\text { various } \\
483(95)\end{array}$ & $\overline{2} 000$ & $\begin{array}{l}21.5 \\
20\end{array}$ & $\begin{array}{l}1 \\
1\end{array}$ & $\begin{array}{l}\text { 85AR } \\
\text { 87LEQ }\end{array}$ \\
\hline
\end{tabular}

\section{Systematic bright star spectroscopy}

This method is obviously very time-consuming although completely safe. It is not surprising that it has only been used in small parts of galaxies, generally in active regions of star formation. See e.g. for giant regions in nearby galaxies, especially M33, d'Odorico et al. (1983) and references herein, and Conti and Massey (1981). The statistics are rarely complete, as more WR stars are generally discovered using other techniques (Drissen 1990). Most of the objects detected in these complexes look overluminous and are probably multiple systems similar to R136 in 30 Dor.

\section{Monochromatic filter imaging}

WR stars are characterised by strong emission lines which are not all the same for the different WR types. It is thus tempting, in order to search systematically for WRs and to separate the WNs from the WCs, to image the galaxy through narrow-band filters on and off emission lines, and then to compare the images either by blinking them or by doing photometry. This technique has been pionneered by Wray and Corso (1972) on M33 using an image-tube with a photographic plate as the detector, then widely used with photographic plates, intensified or not, and with CCDs. Monochromatic 
imaging has definite advantages: high sensitivity, minimum confusion by nearby stars in crowded fields, possibility of distinguishing WCs and WNs by using different filters. The choice of the filter width is rather critical: the best results are obtained with filters whose width matches the width of the lines, i.e. 60-100 A, the reference continuum filters being of similar width (Armandroff and Massey,1985).

This method is unfortunately sensitive to seeing and not completely foolproof. Very red stars can be wrong candidates if the central wavelengths of the filters used in the comparison are rather different, as noted by Moffat and Shara (1987), but this can be avoided. At low levels of detection all sorts of objects can be detected as candidates as shown e.g. by Azzopardi et al. (1988). We agree with Drissen (1990) that only those detections announced with a probability larger than $5 \sigma$ can be considered as reliable. Note also that it is more difficult to detect WNs than WCs as the equivalent widths of their emission lines are generally smaller. Unfortunately we will see later that the luminosity function of WNs extends also to fainter magnitudes than that of WCs, so that the surveys of WCs are always more complete than those of WNs.

Data processing is very important in such work. Usually photometry is secured independently in each color using an appropriate software such as DAOPHOT and the results are compared. If the field is very rich and the background variable as in HII regions, it is probably better to substract directly images obtained with different filters. If the seeing was different there are problems. Drissen (1990) solve them with much success using the CLEAN algorithm applied to each frame. This algorithm has the advantage of giving final images with an arbitrary but identical point-spread function: they can be subtracted with minimal residuals. Presumably this method should be implemented in all future studies.

\section{Field low-resolution spectroscopy}

This method has been used even earlier than the previous one, at least for the Magellanic Clouds. The idea here is to identify the WRs via their emission lines on objective-prism or objective-grating plates. It is very advantageous to use a filter to restrict the spectral range to what is really needed, usually centering in the 4486 A blend. This limits the length of the spectra, hence image crowding in dense regions, and also the sky background. This was done in the late 70's by Azzopardi and Breysacher (see Table 1) for their Magellanic Cloud survey, then for most subsequent surveys. It is commonplace to work on fields as large as 1 square degree using GRISMs or GRENSes at the focus of large telescopes with photographic plates. This is why this method has been so popular until recently. However our own photographic surveys of the extended local group galaxies, especially M33 and M 31 (see Table 1) will probably be the last of the kind.

Field spectroscopy is a relatively safe way of discovering WRs and has the advantage that other types of objects with strong spectral features in the range of the filter can also be discovered, e.g. planetary nebulae or compact HII regions through $\mathrm{H} \beta$ and [OIII] 4959 and $5007 \mathrm{~A}$ emission, or carbon stars through C2 absorption bands with heads at 4735 and $5165 \mathrm{~A}$ : for a discussion see Azzopardi (1989). As with the previous method, it is easier to detect WCs than WNs. Working in the blue-green spectral region, sky background is not a severe problem. Field crowding is not a major problem either if the dispersion is very low, and masking techniques can ease the photographic detection in crowded fields. However the various spectral orders that show up if GRISMs or GRENSes are used make the examination of the images uneas. The use of a simple prism, preferably of Fehrenbach type (no deviation at some central wavelength) is certainly better although it has not yet been implemented on a large telescope. A study made for us at ESO by $\mathrm{H}$. Dekker shows that its use is certainly feasible without difficulty. 
The use of a photographic plate in most previous surveys, while it allows large fields of view, has the drawback of a lack of sensitivity. It has also the usual limitations of limited dynamic range: saturation for bright objects and detection threshold for the continuum of faint objects which appear as points not specific of WRs of course. There is no reason why CCDs cannot be used. We have made recently some experiments with EFOSC at the ESO $3.6 \mathrm{~m}$ telescope (not on WRs unfortunately) which have been very successful. There is no doubt that the sensitivity of the method can be made as good as that of filter imaging provided that the dispersion is chosen such that the size of the WR emission-line spots is limited by seeing, not by dispersion. It will keep the advantage of being more foolproof, while it will probably keep the drawback that building an automatic detection algorithm is not going to be easy. Also, one should look at the possibility of separating the WNs from the WCs, which has not been done up to now.

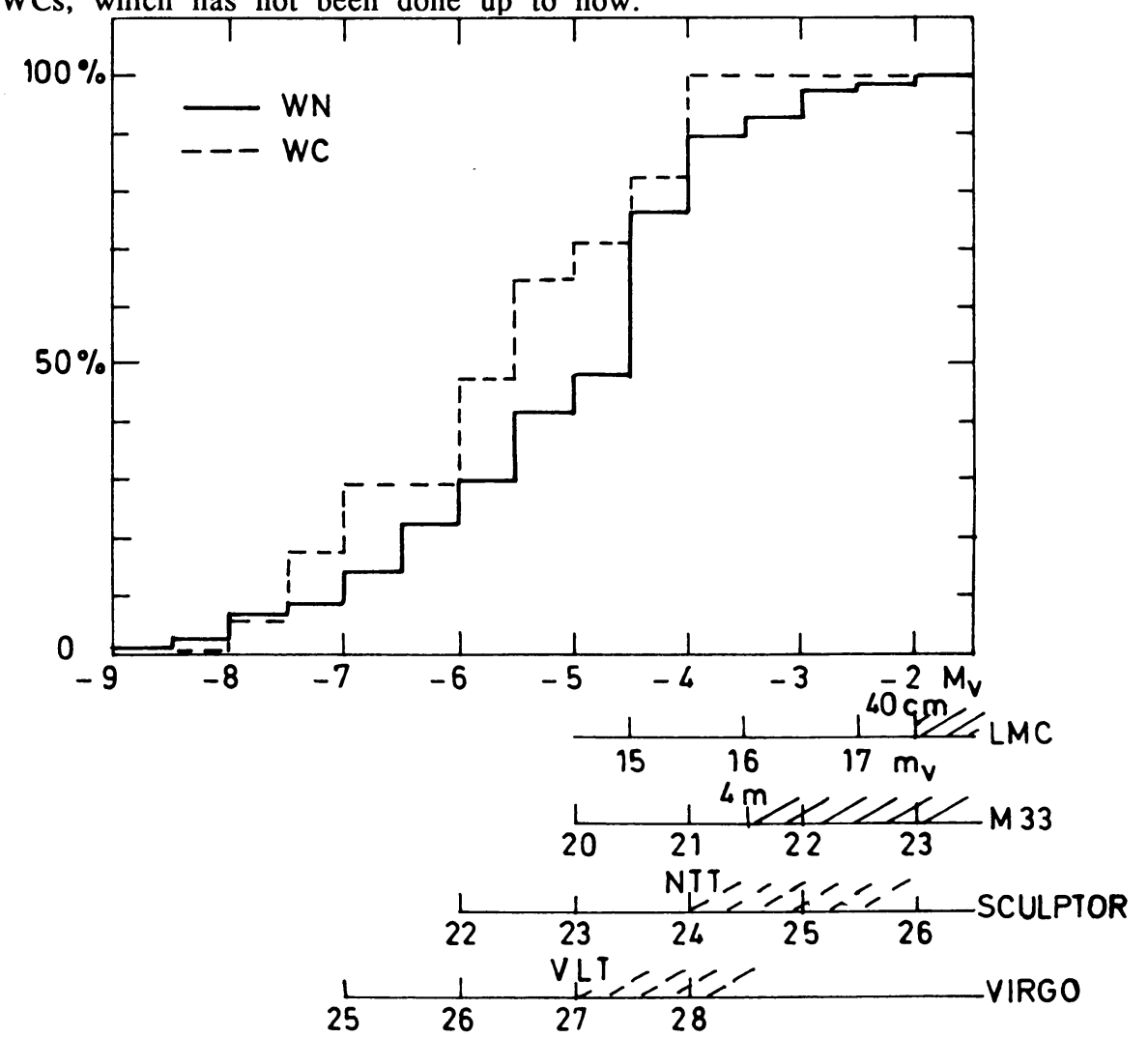

Figure 1. Cumulative distribution of $\mathrm{V}$ absolute magnitudes of WRs of the LMC. Scales at the bottom correspond to galaxies at various distances, taking into account their respective interstellar extinctions. Built from Breysacher (1986).

\section{Completeness of the surveys, present results}

Let us first consider the situation with the Magellanic Clouds. After the completion of the photographic field spectroscopy of Azzopardi and Breysacher there were 104 WRs detected in the LMC and 8 in the SMC. Breysacher (1986) gives visual magnitudes for 94 of the LMC WRs; Fig. 1 displays the cumulative distribution of absolute $\mathrm{V}$ magnitudes for these stars. More powerful subsequent surveys have not detected any new WR in the SMC and only a few more in the LMC: see Table 1; also, 4 new WRs have been 
detected recently by Testor and Schild (1990) and by Heydari-Malayeri et al. (1990). With the exception of 2, these new WRs are in the range of the ones detected with the $40-\mathrm{cm}$ telescope survey. This illustrates the completeness of this survey, and also shows that the distribution of absolute magnitudes given in Fig. 1 can be considered as representative. The limit of $V=17.5$ announced for the survey is also realistic and roughly suffices for completeness. This translates into $V=23$ for $\mathrm{M} 33$, taking into account the average extinction for this galaxy. The published surveys claim a limit of about 21.5. Fig. 1 shows that with this limit only $20 \%$ of the WNs and few WC are missed. It is clear that the photographic survey of Lequeux et al. (1987) which stops at $\mathrm{V}=20$ should be incomplete by about $50 \%$ for the WNs and $20 \%$ for the WCs, in agreement with the figures given by the authors $(55 \%$ and $25 \%$ respectively with respect to surveys limited at $\mathrm{V}=21.5$ ). The situation should be the same for IC1613 and NGC6822 which are roughly at the same distance as M33, although extinction is larger in NGC6822: thus the (small) figures given in Table 1 for these galaxies should reflect approximate completeness and there is little chance to detect other WRs there. The surveys for M31 are certainly much more incomplete although this galaxy is also at the same distance, because of the large internal extinction which is difficult to quantify. However there is no doubt that this galaxy does not contain a large number of WRs, due to its well-known low star-formation activity.

The results of the WR surveys with respect to the WR/O and to the WC/WN ratios have been discussed most recntly by Azzopardi et al. (1988) and in several papers at this Symposium. The surveys of WR stars in giant extragalactic HII regions (Drissen, 1990) show predominently WN features, and give a WR/O star number ratio apparently similar to that in the field of spiral galaxies, while this ratio is expected to be much higher a few $10^{6}$ years after a burst of star formation (the number of $O$ stars can be evaluated as in Lequeux et al. 1981). All this can be explained by clustering of WR stars. If several grouped WR stars are taken as a single star, the spectrum will often be dominated by WN features from late WNs which are the most luminous (see Breysacher, 1986) and the apparent WR/O number ratio will be smaller than the actual ratio.

\section{Perspectives}

Whatever the technique, it is necessary for further studies to secure spectroscopy of the candidates. The most economical way to do so, if one is only interested in the total number of WRs in a field and in the WC/WN ratio, is to use both techniques simultaneously. This is possible in a single observing run with imaging and field spectroscopy devices as EFOSC or EMMI on the ESO telescopes. It should be possible with such an equipment to reach securely a detection limit of about $\mathrm{V}=24$. This would allow an almost complete survey of WRs in galaxies of the Sculptor group (see Fig. 1). With exquisite seeing, e.g. provided by active and adaptative optics, and with the future giant telescopes it might be possible to reach $\mathrm{V}=26$ or 27 and to detect a large fraction of the WRs in the field of galaxies of the Virgo cluster. This is a very exciting perspective indeed!

\section{REFERENCES}

Armandroff, T.E., Massey, P., 1985, Astrophys. J. 291, 685 (85AR)

Azzopardi, M., 1989, in Recent developments of MC research, K.S. de Boer and G. Stasinska, eds. Observatoire de Paris, p. 57

Azzopardi, M., Breysacher, J., 1980, Astron. Astrophys. Suppl. 39, 19 (80AZ)

Azzopardi, M., Breysacher, J., 1979, Astron. Astrophys. 75, 120 (79AZa)

Azzopardi, M., Breysacher, J., 1979, Astron. Astrophys. 75, 243 (79AZb) 
Azzopardi, M., Breysacher, J., 1985, Astron. Astrophys. 149, 213 (85AZ)

Azzopardi, M., Lequeux, J., Maeder, A., 1988, Astron. Astrophys. 189, 34

Bohannan, B., Conti, P.S., Massey, P.,1985, Astron. J., 90, 600 (85BOH)

Breysacher, J., 1986,

Conti, P.S., Massey, P., 1981, Astrophys. J. 249, 471

d'Odorico, S., Rosa, M., Wampler, E.J., 1983, Astron. Astrophys. Suppl. 53, 97

Drissen, L., 1990, Thèse de Doctorat, Université de Montréal (90DRI)

Heydari-Malayeri, M., Melnick, J., van Drom, E., 1990, Astron. Astrophys. in press

Lequeux, J., Meyssonnier, N., Azzopardi, M., 1987, Astron. Astrophys. Suppl. 67,169 (87LEQ)

Lequeux, J., Maucherat-Joubert, M., Deharveng, J.M., Kunth, D., 1981, Astron.

Astrophys. 103, 305

Maeder, A., 1990, Astron. Astrophys. in press

Maeder, A., Lequeux, J. , Azzopardi, M., 1980, Astron. Astrophys. 90, L17-20

Massey, P., Armandroff, T.E., Conti, P.S., 1986, Astron. J., 92, 1303 (86MASa)

Massey, P., Conti, P.S., 1983, Astrophys. J. 273, 576 (83MASb)

Massey, P., Conti, P.S., Moffat, A.F.J., Shara, M.M., 1987, Pub. Astron. Soc. Pac.

99, 816 (87MASb)

Mas-Hesse, J.M., Kunth, D., 1990, in preparation

Meyssonnier, N., Lequeux, J., Azzopardi, M., 1990, in preparation

Moffat, A.F.J., Seggewiss, W., Shara, M.M., 1985, Astrophys. J. 295, 109

$(85 \mathrm{MOFb})$

Moffat, A.F.J., Shara, M.M., 1983, Astrophys. J., 273, 544 (83MOd)

Moffat, A.F.J., Shara, M.M., 1987, Astrophys. J., 320, 266 (87MOFb)

Morgan, D.H., Good, A.R., 1985, Month. Not. R. Astron. Soc. 216, 459 (85MOR)

Morgan, D.H., Good, A.R., 1990, Month. Not. R. Astron. Soc. 243, 459 (90MOR)

Testor, G., Schild, H., 1990, Astron. Astrophys. in press

Westerlund, B.E., Azzopardi, M., Breysacher, J., Lequeux, J., 1983, Astron.

Astrophys. 123, 159 (83WE)

Wray, J.D., Corso, G.J., 1972, Astrophys. J. 172, 577 (72WRA)

\section{DISCUSSION}

Montmerle: There has been some reference to $H S T$ observations of WR stars. I would like to know, perhaps in more detail, what are the current programs for observing with $H S T$ ? Shara: There is a mixture of GTO and regular astronomer time in the first year, and indeed there are some programs to look into $H I I$ regions in M33. So, yes, there is allocated time even in the first year.

Lequeux: The problem with $H S T$ is, it is only a 2.4 meter telescope, so, I think we would do better in the future with a 8 to 10 meter telescope, and the best seeing we can get.

Walborn: The 30 Dor ionizing cluster subtends $3^{\prime}$, which translates into 0.5 at the Virgo cluster distance. This makes detailed studies of WR stars in Virgo $H I I$ regions impossible. 